\title{
Core-scale description of porous media dissolution during acid injection - Part I: Theoretical development
}

\author{
F. GOLFIER ${ }^{1 *}$, B. BAZIN ${ }^{2}$, R. LENORMAND ${ }^{2}$ and M. QUINTARD ${ }^{3}$ \\ ${ }^{1}$ LAEGO, rue du Doyer Marcel Rouboult, BP40, 54501 Vancloeuvre-Pès, Nancy - France \\ ${ }^{2}$ IFP, Avenue de Bois-Préau, 92852 Rueil-Malmaison Cedex - France \\ ${ }^{3}$ Institut de Mécanique des Fluides, Allée C. Soula, 31400 Toulouse - France \\ E-mails: fabrice.golfier@ensg.inpl-nancy.fr / brigitte.bazin@ifp.fr / \\ roland.lenormand@ifp.fr / michel.quintard@imft.fr
}

\begin{abstract}
Dissolution mechanisms in porous media may lead to unstable dissolution fronts ("wormholing"). It has been shown in the literature that Darcy-scale models may reproduce all the characteristics of such dissolution patterns. This paper considers the core-scale averaged behavior of these Darcy-scale dissolution models. The form of core-scale equations is discussed based on the volume averaging of the Darcy-scale equations. The uncertainty about the choice of the unit cell (and boundary conditions) to solve the closure problems and the impact of the dissolution history on the core-scale properties is emphasized.
\end{abstract}

Mathematical subject classification: $76 \mathrm{~V} 05,76 \mathrm{~S} 05,80 \mathrm{~A} 32$.

Key words: core-scale dissolution, one-equation model, porous media, two-equation model, wormhole.

\section{Introduction}

Dissolution mechanisms in porous media are frequently encountered in stimulation of petroleum wells [37], or more generally in hydrogeology [17]. Stimulation methods consist in injecting an acid solution into the formation in order to increase the rock permeability. The dissolution process develops preferentially in the largest pores which leads to the formation of highly conductive flow

\#572/03. Received: 24/IV/03. Accepted: 04/III/04.

*Corresponding author. 
channels called wormholes. As a consequence, prediction of wormhole propagation and the understanding of dissolution regimes present a major interest to optimize acidizing treatments. However, the description of dissolution macroscopic patterns is a very complex problem, especially at the large-scale, since it is determined by the flow microscopic characteristics, and with many factors in play such as the injection rate, the acid volume, and rock permeability.

At the pore-scale, the dissolution mechanism can be separated into three types: the acid transport by diffusion and advection to the solid surface, the chemical reaction at the surface and the product transport away from the surface. Two limit cases can be considered. If the chemical reaction characteristic time is very short compared to the mass-transfer kinetics, the reaction is called mass-transferlimited. This is generally the case for limestone dissolution with $\mathrm{HCl}$ as verified by Lund et al. [25]. If mass-transfer kinetics is slow, then the reaction is reactionrate limited. It is the case for dolomite at the room temperature as recognized by Lund et al. [24]. It must be emphasized here that these pore-scale dissolution mechanisms must not be confused with the macroscopic or Darcy-scale condition of local equilibrium or local non-equilibrium. Local-equilibrium dissolution corresponds to a macro-scale acid concentration equal to the micro-scale equilibrium concentration over the solid interface. This results into immediate consumption of the acid at the entrance of the porous medium, and this will lead to a sharp interface between the fluid zone and the porous medium. On the opposite, local non-equilibrium dissolution will lead to a dissolution zone within the porous domain characterized by a variable porosity. This dissolution process is coupled with the fluid momentum equation in an unstable way: flow velocity is higher in the largest pores, which will generally produce faster dissolution processes. These processes increase locally the pore diameter and this may in turn facilitate the acid transport to these large pores. These physical mechanisms lead to very complicated macro-scale dissolution patterns as described below.

Several experimental core tests $[18,7,40,2,9,11,15]$ have been performed to understand the physics of this dissolution phenomenon. Five dissolution regimes, illustrated in Figure 1 for salt dissolution [15], have been observed experimentally depending on the flow rate $[18,7,11]$ as explained below:

- (a) Face or compact dissolution: At low injection rate, the acid is com- 
(a)

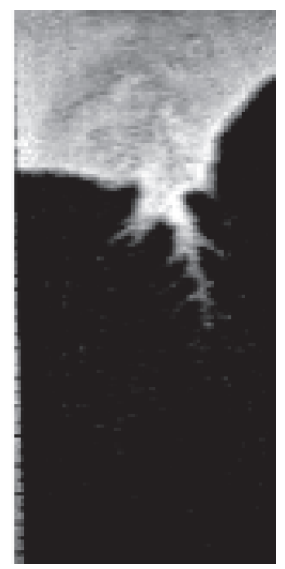

(b)

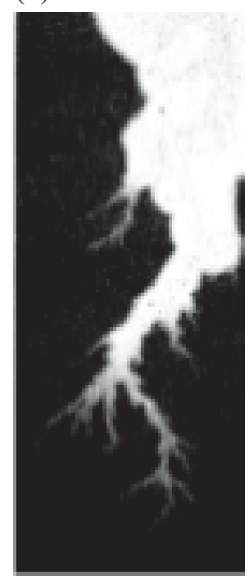

(c)

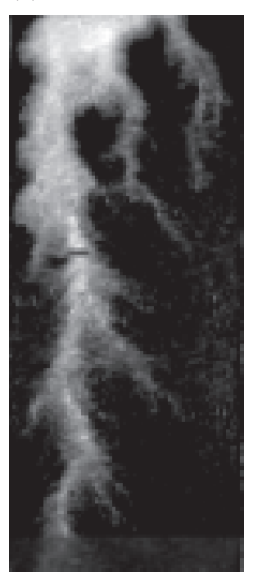

(d)

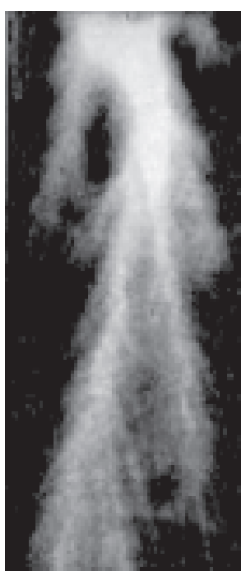

Figure 1 - Example of experimental patterns for salt dissolution: (a) face dissolution, $Q=5 \mathrm{~cm}^{3} \cdot \mathrm{h}^{-1}, C_{N a C l}=150 \mathrm{~g} \cdot \mathrm{l}^{-1}$; (b) conical wormhole, $Q=10 \mathrm{~cm}^{3} \cdot \mathrm{h}^{-1}$, $C_{\mathrm{NaCl}}=230 \mathrm{~g} . \mathrm{l}^{-1}$; (c) dominant wormhole, $Q=50 \mathrm{~cm}^{3} \cdot \mathrm{h}^{-1}, C_{\mathrm{NaCl}}=150 \mathrm{~g} . \mathrm{l}^{-1}$; (d) ramified wormhole, $Q=250 \mathrm{~cm}^{3} \cdot \mathrm{h}^{-1}, C_{N a C l}=150 \mathrm{~g} .1^{-1}$ (Golfier et al., J. Fluid Mech., 2002).

pletely consumed at the inlet of the core. Diffusion mechanism predominates over transport by convection, instabilities cannot develop. This regime corresponds to local equilibrium dissolution.

- (b) Conical wormholes: By increasing slightly the injection rate, instabilities begin to develop although the regime is still strongly influenced by diffusion. The reactant starts to penetrate in the medium and erodes the walls of the flow channels, to form a conical-shaped wormhole. This regime is also characterized by local equilibrium dissolution.

- (c) Dominant wormholes: At intermediate flow rates, acid penetrates preferentially into the biggest pores to form flow channels. Eventually, this leads to the formation of a dominant wormhole. The optimum flow rate, defined as the flow rate that produces the longest wormhole for the minimum acid consumption is found in this regime. This regime is also characterized by local equilibrium dissolution. 
- (d) Ramified wormholes: At high injection rate, local non-equilibrium dissolution starts to occur. The wormholes become more highly branched or ramified and the dissolution front is more spread within the porous domain.

- (e) Uniform dissolution: At last, at very high injection rate, the reactant is forced into all pores in the porous matrix. The dissolution front is spread all over the core length, and this corresponds to a non-equilibrium dissolution. This latter regime could not be reached for the salt dissolution due to the use of an unconsolidated porous material.

Besides the dissolution patterns, another interesting feature has been demonstrated experimentally. It is now fully evidenced that the pore acid volume injected to breakthrough the core depends on the injection rate and goes through a minimum at the optimum injection rate $[18,40,2]$. It is reported that the optimum conditions are related to the formation of a dominant wormhole with little branching through the core and depends on several parameters, including the rock mineralogy (calcite or dolomite for carbonate formations), the temperature and the acid concentration. They have been worked out in detail by Bazin and Abdulahad [1].

In order to predict this optimum injection rate and the wormhole development at the Darcy-scale, several numerical models have been developed. Among the different approaches proposed in the literature, we can cite the works of Hoefner and Fogler [18], Wang et al. [40], Daccord et al. [7], Liu et al. [23] or Fredd and Fogler [10]. The reader can refer to the paper by Fredd and Miller [12] for a detailed presentation of these different models. Since none of these models could describe all the features of the dissolution physics, in particular they failed to describe the coupled nature of flow and reaction without assuming a wormhole geometry, a macroscopic acid-transport model has been implemented based on a macro-scale description involving a non-equilibrium mass balance model [15]. Concerning the momentum equation, assuming that the velocity of the pore-scale interfaces is small enough, a Darcy-Brinkman formulation has been adopted that allows to simulate correctly the flow in the fluid or porous zones in a continuous manner $[39,3,38]$. The effect of dissolution history on the effective properties, such as the permeability, has been approximated by direct relationships between 
the macro-scale parameters. For instance, a direct relationship is adopted between the permeability and the porosity, which is a classical assumption made in geochemistry.

A comparison of the numerical simulations obtained with this model with experimental results showed a good agreement in terms of dissolution regimes and optimum flow rate. However, a direct application of laboratory results to the field scale is not straightforward since a direct Darcy-scale description would require a very fine grid. Therefore, a large-scale model is necessary. Two different approaches able to fit the wormhole propagation models at the core-scale can be distinguished in the literature. The first one used by Daccord et al. [7] or Huang et al. [19] consists to fit the injection flow rate with a surface area scaling method in order to keep the same injection velocity into the porous matrix. Nevertheless, this approach needs that the wormhole density is the same whatever the observed scale. This assumption has some important consequences on the modelling of wormhole competition at the field-scale and leads to overestimate the number of wormholes created per surface unit. The calculation of fluid loss at the walls may be modified if necessary to take into account the new geometry of the problem (radial instead of linear). The second approach, developed by Huang et al. [20] and Fredd [8] consists to fit the injection flow rate from the number of wormholes by keeping the same reaction and transport rates for all of them. The drawback of this method is that it requires a predictive model of wormhole density. Several studies have tried to solve this problem. Huang et al. [20] used a capillary tube model to determine the minimal value of pressure perturbations necessary to make appear a new wormhole. Gdanski [14] based his model on some assumptions of symmetry and geometry. Fredd [8] used the results of Hoefner and Fogler [18] and Buijse [4] linking the number of created wormholes to the ratio of the inlet area on the given propagation length. It must be emphasised that this last assumption is based only on some numerical simulations (capillary tube or network model) and has been exploited by Fredd [8] for all the dissolution regimes, even if it concerns only the wormholing regime. These different models are generally used to estimate the skin effect factor [13], which corresponds in a field model to a parameter modifying the transfer between the well and the field, in order to take into account the real conditions 
around the well. Nevertheless, these conditions are limited to some simplified cases (homogeneous medium, vertical wells). The reader can refer to Fredd and Miller [12] for a comparison of the models cited above. This discussion emphasizes the difficulties to adapt the existing dissolution models at the field-scale. We expect from an upscaling technique such as the volume averaging method an improvement in the modelling, by a better incorporation of large-scale physics. Nevertheless, the upscaling to the field-scale is not straightforward. We believe that it is necessary to study an intermediate upscaling corresponding to the corescale, represented Figure 2. Such an approach belongs to the class of averaged models, which, to our knowledge, has not yet been applied to the wormholing phenomenon. This model is however frequently used to model the viscous fingering [21, 41], even if in our case, the problem is a little bit more complex since the acid concentration is defined in both regions, wormhole and porous medium.

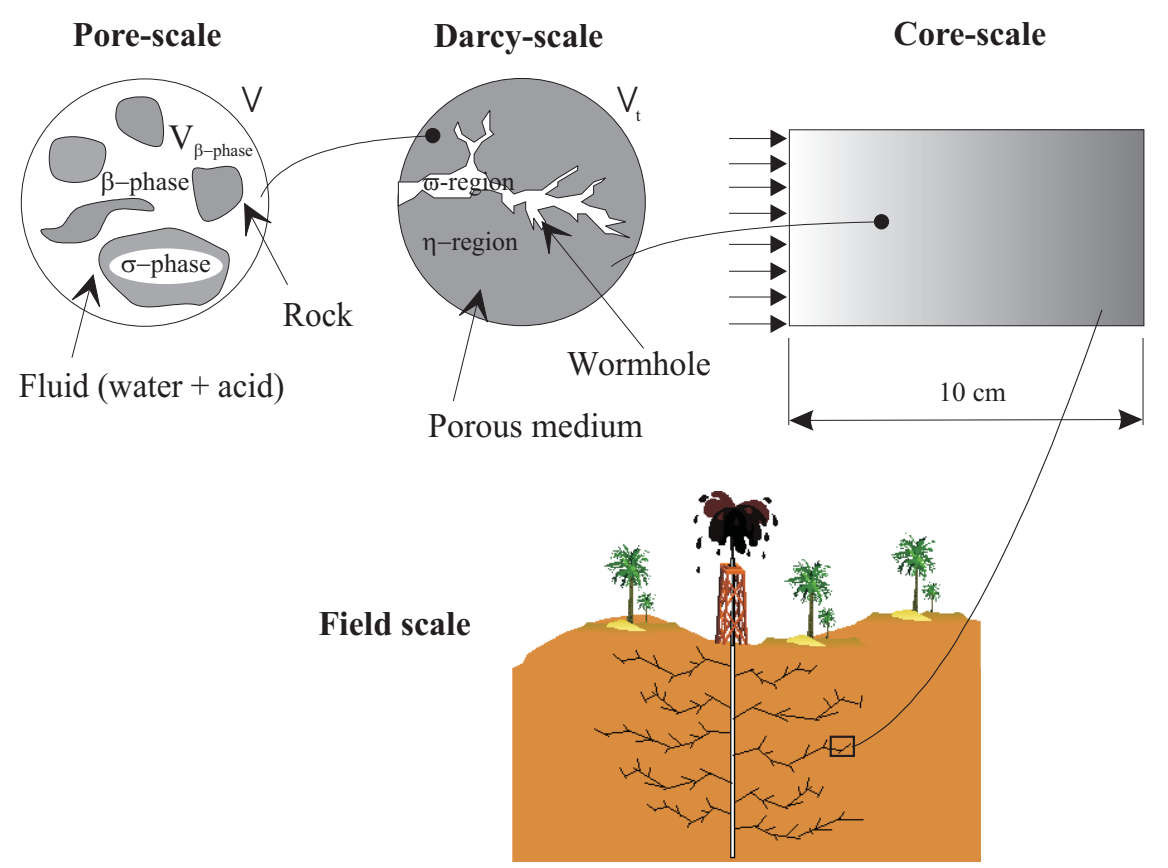

Figure 2 - The different scales of the problem.

In this paper, we consider the core-scale, and look at the theoretical development of macroscopic relations that could be used in well modeling. The idea 
is to start with a local-scale model able to reproduce the wormholing process. Such a model has been described in Golfier et al. [15] and we briefly summarize the introduction of this model since it has some analogy with the upper-scale problem. The possible structure of a core-scale model is discussed, especially in terms of the form of the equations.

\section{Darcy-scale model}

In Golfier et al. [15], we have obtained wormhole patterns by using a continuous Darcy-scale description of the flow in the dissolved region or in the porous domain. The dissolution macroscopic equations were obtained from the coupled equations of flow and species transport at the pore-scale, and this led to a local non-equilibrium dissolution problem. Most of the theoretical questions associated with this development have been discussed in Quintard and Whitaker [36]. The solid phase and the fluid phase are identified as the $\sigma$-phase and the $\beta$-phase respectively. In order to be clear about the equations we have used, we will follow the notations associated with the volume averaging theory presented in Quintard and Whitaker [36]. For instance, the superficial velocity is given by

$$
\mathbf{V}_{\beta}=\left\langle\mathbf{v}_{\beta}\right\rangle=\frac{1}{V} \int_{V_{\beta}} \mathbf{v}_{\beta} d V
$$

where $V_{\beta}$ represents the volume of the $\beta$-phase contained within the averaging volume, $V$, as illustrated in figure 2 . The intrinsic average pressure and mass fraction are defined by

$$
\begin{gathered}
P_{\beta}=\left\langle p_{\beta}\right\rangle^{\beta}=\frac{1}{V_{\beta}} \int_{V_{\beta}} p_{\beta} d V \\
C_{A \beta}=\left\langle c_{A \beta}\right\rangle^{\beta}=\frac{1}{V_{\beta}} \int_{V_{\beta}} c_{A \beta} d V
\end{gathered}
$$

We just consider here that the reaction is mass-transfer limited, and the acid is immediately consumed as soon as he reacts with the solid. In addition, the additional convective terms which appear during the upscaling are neglected. By this way, we obtain a complete system of equations which combines a DarcyBrinkman model for a rigorous description of the flow in both zones (fluid and 
porous region) with a local non-equilibrium dissolution model. It can be written as follows:

\section{Flow equations:}

$$
\begin{gathered}
\frac{\mu}{\varepsilon_{\beta}} \Delta \mathbf{V}_{\beta}-\left(\nabla P_{\beta}-\rho_{\beta} \mathbf{g}\right)-\mu \mathbf{K}^{-1} \cdot \mathbf{V}_{\beta}=0 \\
\nabla \cdot \mathbf{V}_{\beta}=0
\end{gathered}
$$

\section{Species transport equations:}

$$
\begin{gathered}
\varepsilon_{\beta} \frac{\partial C_{A \beta}}{\partial t}+\mathbf{V}_{\beta} \cdot \nabla C_{A \beta}=\nabla \cdot\left(\mathbf{D}_{\beta}^{*} \cdot \nabla C_{A \beta}\right)-\alpha C_{A \beta} \\
\frac{\partial \varepsilon_{\beta}}{\partial t}=\frac{\beta}{\rho_{\sigma}} \alpha C_{A \beta}
\end{gathered}
$$

where $\mu$ represents the viscosity, $\beta$ the stoichiometric coefficient of the reaction, $\mathbf{K}$ the permeability tensor and $\varepsilon_{\beta}$ the porosity. We denote also the fluid and rock density as $\rho_{\beta}$ and $\rho_{\sigma}$ respectively. The effective coefficients which appear in the transport equations, the mass transfer coefficient $\alpha$ and the dispersive tensor $\mathbf{D}_{\beta}^{*}$, are calculated at each node for a given porosity and velocity field from some numerical simulations at the pore-scale on a representative periodic unit cell (use of the closure problems described in Golfier et al. [15]). The permeability field is directly linked with the porosity, by the classical Kozeny-Carman relation $K(\varepsilon)$.

A numerical model has been developed to solve these equations, all the details can be found in Golfier et al. [15]. Various calculations have been performed on two-dimensional domains. In particular, they allowed to capture all the dissolution regimes and confirmed the existence of an optimum injection rate. As suggested by the different obtained results, the proposed Darcy-scale dissolution model shows a good agreement with laboratory experimental results. However, because of heavy computational requirements, similar numerical results cannot be directly obtained for a larger geometry, such as the one necessary for well models. Therefore, there is a need for a larger-scale model, and this is discussed in the next section. 


\section{Core-scale results and discussion}

In this section, macroscopic equations which describe the problem at the corescale are first presented. Arguments are provided indicating that a classical Darcy model may be used for the flow description at the upper scale, and that the form of the mass transport macroscopic equation depends on the mass transfer coefficient value. The uncertainty about the choice of the unit cell (and boundary conditions) to solve the closure problems and the impact of the dissolution history on the core-scale properties is emphasized.

\subsection{Core-scale flow equation}

The core-scale flow equation developed here is based on the works of Quintard [27] and Lasseux et al. [22]. From notations presented in Quintard and Whitaker [35], core-scale averaged quantities associated with the core-scale averaging volume $V_{t}$ described in Figure 2 are defined as

$$
\begin{aligned}
& \mathbf{V}_{\beta}^{*}=\frac{1}{V_{t}} \int_{V_{t}} \mathbf{V}_{\beta} d V \\
& P_{\beta}^{*}=\frac{1}{V_{t}} \int_{V_{t}} P_{\beta} d V
\end{aligned}
$$

With this definition, the average of Eq. (5) leads simply to

$$
\nabla \cdot \mathbf{V}_{\beta}^{*}=0
$$

In order to apply the volume averaging technique to the Darcy-Brinkman equation, Eq. (4), we introduce the spatial deviation associated to the local parameter $P_{\beta}$ following Gray's decomposition [16], we have

$$
P_{\beta}=P_{\beta}^{*}+\tilde{P}_{\beta}
$$

Introducing this decomposition into Eq. (4), we get

$$
\frac{\mu_{\beta}}{\varepsilon_{\beta}} \Delta \mathbf{V}_{\beta}-\nabla \tilde{P}_{\beta}-\left(\nabla P_{\beta}^{*}-\rho_{\beta} \mathbf{g}\right)+\mu_{\beta} \mathbf{K}^{-1} \cdot \mathbf{V}_{\beta}=0
$$

The underlying idea is that, the equations being linear, a direct relationship between the velocity and the gradient of the averaged pressure over the representative unit cell can be obtained. As a consequence, it is necessary to express the 
macroscopic properties and the spatial deviations from the mapping variables $\mathbf{B}$ and $\mathbf{b}$ under the form

$$
\begin{gathered}
\mathbf{V}_{\beta}=-\frac{1}{\mu_{\beta}} \mathbf{B} \cdot\left(\nabla P_{\beta}^{*}-\rho_{\beta} \mathbf{g}\right) \\
\tilde{P}_{\beta}=\mathbf{b} \cdot\left(\nabla P_{\beta}^{*}-\rho_{\beta} \mathbf{g}\right)
\end{gathered}
$$

Introducing these representations into Eq. (10) and (12), we obtain the following closure problem, which provides explicit relationships between the local structure and the core-scale equations.

\section{Problem I:}

$$
\begin{aligned}
& \frac{1}{\varepsilon_{\beta}} \Delta \mathbf{B}-\mathbf{K}^{-1} \cdot \mathbf{B}=\nabla \mathbf{b}+\mathbf{I} \\
& \nabla \cdot \mathbf{B}=0 \\
& \frac{1}{V_{t}} \int_{V_{t}} \mathbf{b} d V=0 \\
&+ \text { B.C. (periodicity,.. ) }
\end{aligned}
$$

From the dissolution patterns presented in the previous paragraphs, it is obvious that this is not a simple matter to define a unit cell representative of the medium geometry at the local-scale as it is classically done for the closure problems at the pore-scale. In particular, questions arise about the use of boundary conditions for the mapping variables $\mathbf{b}$ and $\mathbf{B}$. Periodicity may not be used in Eq. (15d), but we leave open this problem at this time. It seems even more difficult to introduce the time evolution of the unit cell geometry or the impact of the dissolution history on the effective properties. We have solved this problem at the porescale by assuming that the dissolution propagated in a uniform way. However, this assumption is not consistent at the core-scale with the propagation mode of the wormholes. These difficulties will appear for every closure problem in this paper, and we will come back to this point in Part II.

Now, taking the average of Eq. (13) we obtain the following averaged equation

$$
\mathbf{V}_{\beta}^{*}=\frac{1}{V_{t}} \int_{V_{t}} \mathbf{V}_{\beta} d V=-\frac{1}{\mu_{\beta}} \mathbf{K}^{*} \cdot\left(\nabla P_{\beta}^{*}-\rho_{\beta} \mathbf{g}\right)
$$


where the effective core-scale permeability tensor is given by

$$
\mathbf{K}^{*}=\frac{1}{V_{t}} \int_{V_{t}} \mathbf{B} d V
$$

This development suggests that the core-scale equations have still the form of a Darcy equation, the effective permeability being determined by Problem I. It must be emphasized that the underlying assumption in this development is that the time evolution of the dissolved region is slow enough so that the pressure field relaxation occurs immediately. The wormhole interface is quasi-steady with respect to the momentum equation. However, the core-scale permeability tensor, $\mathbf{K}^{*}$, becomes time dependent. Like in the Darcy-scale model, the effective parameters depend a priori upon the dissolution history. At this point, the question is left open whether it is possible to replace this dissolution history effect by a direct relationship with a core-scale parameter similar to the Darcy-scale porosity or not.

However, even if the flow can be described by a one-equation model based on the validity of core-scale mechanical equilibrium, it may be necessary under some circumstances to determine the regional velocities [35]. This occurs when core-scale mass equilibrium is not valid and a two-equation model is required to describe the process of convection, dispersion and reaction. These regional average velocities, $\mathbf{V}_{\varpi}^{*}$ and $\mathbf{V}_{\eta}^{*}$, are linked to the average velocity $\mathbf{V}_{\beta}^{*}$ by the following relationship

$$
\mathbf{V}_{\beta}^{*}=\mathbf{V}_{\varpi}^{*}+\mathbf{V}_{\eta}^{*}
$$

and are solutions of the regional forms of Darcy's law described in Quintard and Whitaker [35]:

$$
\begin{aligned}
\nabla \cdot \mathbf{V}_{\varpi}^{*} & =0 \\
\mathbf{V}_{\varpi}^{*} & =-\frac{1}{\mu} \mathbf{K}_{\varpi}^{*} \cdot\left(\nabla P_{\varpi}^{*}-\rho_{\beta} \mathbf{g}\right) \quad \text { in the } \varpi-\text { phase }
\end{aligned}
$$

and

$$
\begin{aligned}
\nabla \cdot \mathbf{V}_{\eta}^{*} & =0 \\
\mathbf{V}_{\eta}^{*} & =-\frac{1}{\mu} \mathbf{K}_{\eta}^{*} \cdot\left(\nabla P_{\eta}^{*}-\rho_{\beta} \mathbf{g}\right) \quad \text { in the } \eta-\text { phase }
\end{aligned}
$$


where $\mathbf{K}_{\varpi}^{*}, \mathbf{K}_{\eta}^{*}, P_{\varpi}^{*}$ and $P_{\eta}^{*}$ represent the regional permeability tensors and pressures.

\subsection{Core-scale transport and dissolution equation}

With regard to the transport and dissolution part, it is not obvious to apply an upscaling method which leads to core-scale equations valid in a general way. In fact, the mass transfer coefficient value can strongly modify the acid transport behaviour and the corresponding Darcy-scale dissolution pattern. This upscaling problem embodies all the difficulties associated with upscaling: potential for abnormal dispersion, non-local effects in space and time. Different perspectives may be opened to deal with such problems. In this paper, we propose, as a preliminary approach, two different models based on the consideration that this is a special case of a two-medium problem.

\subsubsection{Local mass equilibrium dissolution (Da large): two-medium or two-equation model}

Although the Darcy-scale equations have been written under an assumption of local mass non-equilibrium, if the interfacial exchange flux is very important versus the convective flux (dissolution regimes (a), (b) and (c) of Figure 1), the problem leads to the limit case of local mass equilibrium dissolution. To be more precise, if $\alpha \rightarrow \infty$, the Darcy-scale problem can be simplified as follows

$$
\begin{gathered}
\frac{\partial C_{A \beta}}{\partial t}+\nabla \cdot\left(\mathbf{V}_{\beta} C_{A \beta}\right)=\nabla \cdot\left(D \nabla C_{A \beta}\right) \text { in } V_{\varpi} \\
\frac{\partial \rho_{\beta}}{\partial t}+\nabla \cdot\left(\mathbf{V}_{\beta} \rho_{\beta}\right)=0 \text { in } V_{\varpi} \\
C_{A \beta}=0 \text { in } V_{\eta} \\
\frac{\partial \rho_{\eta}}{\partial t}=0 \text { in } V_{\eta}
\end{gathered}
$$

B.C.1:

$$
C_{A \beta}=0 \text { at } A_{\varpi \eta}
$$


B.C.2:

$$
\begin{aligned}
-\rho_{\eta} \mathbf{w} \cdot \mathbf{n}_{\varpi \eta} & =C_{\sigma \beta}\left(\mathbf{V}_{\sigma \beta}-\mathbf{w}\right) \cdot \mathbf{n}_{\varpi \eta} \text { at } A_{\varpi \eta} \\
& =-\beta^{*} C_{A \beta}\left(\mathbf{V}_{A \beta}-\mathbf{w}\right) \cdot \mathbf{n}_{\varpi \eta}
\end{aligned}
$$

where $\rho_{\eta}$ represents the porous medium density, i.e., $\varepsilon_{\sigma} \rho_{\sigma}$, and $\beta^{*}$ the stoichiometric coefficient for the dissolution of the porous medium. This problem is reminiscent of the pore-scale problem that led to Eq. (6) and to the local mass non-equilibrium model [15]. In this particular case, if we follow the same ideas, we would get a two-equation model taking into account the physical mechanisms characteristics of each region. This can be viewed in the framework of two-region, or two-porosity models. There has been an extensive literature on the subject, and we comment below some major features. Considering dispersion mechanisms on a broader sense, two types of situations have been considered in the literature: mobile-immobile models (see Zhang and Smith [41] for an illustration of this idea to the problem of viscous fingering for example) or mobile-mobile models (see a review of this problem in Cherblanc et al. [6]). The most permeable medium, where advection and dispersion mechanisms are preponderant, is called mobile, whereas the less permeable medium, where the diffusion prevails, is considered immobile. Thereafter, these models have been generalised for mobile-mobile systems, i.e., systems for which the advection occurs also in the least permeable region.

Referring to the averaging volume illustrated in Figure 2, we define, for a function $\langle\Psi\rangle$ associated with the $\varpi$-region, the regional average, Eq. (30), and the intrinsic regional average, Eq. (31), according to

$$
\begin{gathered}
\{\langle\Psi\rangle\}_{\varpi}=\frac{1}{V_{t}} \int_{V_{\varpi}}\langle\Psi\rangle d V \\
\{\langle\Psi\rangle\}_{\varpi}^{\varpi}=\frac{1}{V_{\varpi}} \int_{V_{\varpi}}\langle\Psi\rangle d V
\end{gathered}
$$

and both averages are linked by the following relation

$$
\{\langle\Psi\rangle\}_{\varpi}=\phi_{\varpi}\{\langle\Psi\rangle\}_{\varpi}^{\varpi}
$$

where $\phi_{\varpi}=V_{\varpi} / V_{t}$ represents the volume fraction of the $\varpi$-region. Similar definitions hold for the $\eta$-region. Moreover, the averaging volume for the twoequation model is always homogeneous (there is no variation of porosity or 
permeability in the porous medium since we are in local equilibrium). Following the development used for the Darcy-scale advection-reaction equations [15] and based on the same assumption of quasi-stationarity, a first approximation of the problem leads to the following core-scale model

$$
\begin{aligned}
\phi_{\varpi} \frac{\partial C_{A \varpi}^{*}}{\partial t}+\mathbf{V}_{\varpi}^{*} \cdot \nabla C_{A \varpi}^{*} & =\nabla \cdot\left(\mathbf{D}_{\varpi}^{* *} \cdot \nabla C_{A \varpi}^{*}\right)-\alpha^{*} C_{A \varpi}^{*} \\
\frac{\partial \phi_{\varpi}}{\partial t} & =\frac{\beta^{*}}{\rho_{\eta}} \alpha^{*} C_{A \varpi}^{*} \\
& =\frac{\beta}{\rho_{\sigma}} \alpha^{*} C_{A \varpi}^{*} \\
C_{A \eta}^{*} & =0
\end{aligned}
$$

which is mathematically equivalent to the form of the equations previously obtained at the Darcy-scale, but now for a core-scale porosity corresponding to the dissolved region. The core-scale averaged quantities, core-scale intrinsic acid concentration $C_{A \varpi}^{*}$ and superficial velocity $\mathbf{V}_{\varpi}^{*}$, are defined as follows

$$
\begin{array}{r}
C_{A \varpi}^{*}=\left\{C_{A \beta}\right\}_{\varpi}^{\varpi}=\frac{1}{V_{\varpi}} \int_{V_{\varpi}} C_{A \beta} d V \\
\mathbf{V}_{\varpi}^{*}=\left\{\mathbf{V}_{\beta}\right\}_{\varpi}=\frac{1}{V_{t}} \int_{V_{\varpi}} \mathbf{V}_{\beta} d V
\end{array}
$$

We have now to determine the local closure problems in order to couple both scales and calculate the effective coefficients. The general idea, as for the lowerscale, is based on Gray's decomposition [16], which is used to express the local properties as a function of averaged quantities and spatial deviations, i.e.,

$$
\begin{aligned}
C_{A \beta} & =C_{A \varpi}^{*}+\tilde{C}_{A \beta} \\
\mathbf{V}_{\beta} & =\left(\phi_{\varpi}\right)^{-1} \mathbf{V}_{\varpi}^{*}+\tilde{\mathbf{V}}_{\beta}
\end{aligned}
$$

Following the development in Quintard and Whitaker [36], it is possible to approximate the spatial deviation concentration $\tilde{C}_{A \varpi}$ as follows

$$
\tilde{C}_{A \beta}=\mathbf{b}_{\varpi} \cdot \nabla C_{A \varpi}^{*}-s_{\varpi} C_{A \varpi}^{*}
$$


where the mapping variables, $\mathbf{b}_{\varpi}$ and $s_{\varpi}$, are defined by two so-called closure problems, as described below.

\section{Problem II a:}

$$
\begin{aligned}
\tilde{\mathbf{V}}_{\beta}+\mathbf{V}_{\beta} \cdot \nabla \mathbf{b}_{\varpi}= & \nabla \cdot\left(D \nabla \mathbf{b}_{\varpi}\right)-\left(\phi_{\varpi}\right)^{-1} \mathbf{u}_{\varpi} \\
\text { B.C.1 } \quad \mathbf{b}_{\varpi}= & 0 \text { at } A_{w \eta} \\
& + \text { B.C. }(\text { periodicity, ...) } \\
\left\{\mathbf{b}_{\varpi}\right\}_{\varpi}^{\varpi}= & 0
\end{aligned}
$$

where $\mathbf{u}_{\beta}$ in Eq. (41a) is given by:

$$
\mathbf{u}_{\varpi}=\frac{1}{V_{t}} \int_{A_{w \eta}} \mathbf{n}_{w \eta} \cdot\left(D \nabla \mathbf{b}_{\varpi}\right) d A
$$

\section{Problem II b:}

$$
\begin{aligned}
& \mathbf{V}_{\beta} \cdot \nabla s_{\varpi}=\nabla \cdot\left(D \nabla s_{\varpi}\right)-\left(\phi_{\varpi}\right)^{-1} \alpha^{*} \\
& \text { B.C.1 } s_{\varpi}=1 \text { at } A_{w \eta} \\
& \text { + B.C. (periodicity, ...) } \\
& \left\{s_{\varpi}\right\}_{\varpi}^{\varpi}=0
\end{aligned}
$$

where the mass transfer coefficient $\alpha^{*}$, Eq. (43a), is defined by

$$
\alpha^{*}=\frac{1}{V_{t}} \int_{A_{w \eta}} \mathbf{n}_{w \eta} \cdot\left(D \nabla s_{\varpi}\right) d A
$$

whereas the large-scale dispersion tensor $\mathbf{D}_{\omega}^{* *}$ is expressed as

$$
\mathbf{D}_{\varpi}^{* *}=D\left(\phi_{\varpi} \mathbf{I}+\frac{1}{V_{t}} \int_{A_{w \eta}} \mathbf{n}_{w \eta} \mathbf{b}_{\varpi} d A\right)-\left\{\widetilde{\mathbf{V}}_{\beta} \mathbf{b}_{\varpi}\right\}
$$

As explained in Quintard and Whitaker [32], this result requires that the velocity of the interface between the fluid region and the porous region (the solid region in the original pore-scale problem) is small enough so that the concentration field relaxes more rapidly. We are confronted also to the problem of the use of periodicity conditions or other boundary conditions in our closure 
problems (and to the choice of the cell geometry also). Furthermore, like for the permeability, the effective parameters are dissolution history dependent. Is it possible to replace this dependence by a simple unique relationship with the other core-scale parameters? This very important question will be examined in detail in Part II.

\subsubsection{Local mass non-equilibrium dissolution ( $D$ a small): one-medium or one-equation model}

Under local non-equilibrium conditions at the Darcy-scale, the more complex form of the local equations do not allow to directly infer the core-scale transport equation form by keeping the coupling of the scales through the closure problems. In fact, if we apply the volume averaging method to the generalised transport equation, the non-linear term $\alpha C_{A \beta}$ where the two coefficients depend on the spatial coordinates and the time parameter, prevent us from obtaining an averaged equation in a simple manner. Nevertheless, it is possible to define a general form for the macroscopic transport-reaction equations within a one-equation model. A few precise details must be given here about the choice of this model. Different kinds of one-equation models are classically used in the literature. One can distinguish the ones, based on the core-scale local mass equilibrium assumption $[31,34]$, characterised by the following relation,

$$
\left\{C_{A \beta}\right\}_{\varpi}^{\varpi}=\left\{C_{A \beta}\right\}_{\eta}^{\eta}=\left\{C_{A \beta}\right\}
$$

It is then very simple, by adding the equations of each region of the twoequation model, to obtain a local equilibrium one-equation model. Unfortunately, local equilibrium conditions are difficult to be applied at the scale that we study. In fact, what is the physical significance of a core-scale local mass equilibrium? If local equilibrium conditions exist at the Darcy-scale, which can be expressed by the relation $C_{A \beta}=0$ in the $\eta$-phase, this means that $\left\{C_{A \beta}\right\}_{\varpi}^{\sigma}=\left\{C_{A \beta}\right\}_{\eta}^{\eta}=0$, i.e., that the acid concentration is also equal to zero in the wormholes. If this assumption was acceptable at the lower scale because of the small pore size, it seems more difficult to be verified here because of the wormhole size, and, as a consequence, the minor importance of diffusive effects. 
From this discussion, we have chosen a model which does not need such an assumption, a one-medium or local non-equilibrium one-equation model, based on the works of Moyne et al. [26], Cherblanc [5] and Quintard et al. [28].

Macroscopic averages are defined in the $V_{t}$ volume. The core-scale porosity is given by

$$
\varepsilon_{\beta}^{*}=\frac{1}{V_{t}} \int_{V_{t}} \varepsilon_{\beta} d V
$$

Here some comments must be introduced about the two parameters $\varepsilon_{\beta}^{*}$ and $\phi_{\varpi}$. This latter parameter plays the role of $\varepsilon_{\beta}$ at the Darcy-scale. If dissolution occurs as a local equilibrium phenomenon, there is a direct relationship between $\varepsilon_{\beta}^{*}$ and $\phi_{\varpi}$, we have

$$
\varepsilon_{\beta}^{*}=\phi_{\varpi}+\left(1-\phi_{\varpi}\right) \varepsilon_{\beta}
$$

and any of these two parameters can be used to describe the system. Such a relationship does not hold in the case of local non-equilibrium dissolution, and, as a consequence, the two parameters are somehow independent while linked by the dissolution history.

The macroscopic concentration $C_{A \beta}^{*}$ is obtained from an average weighted by the porosity. As in the case of two-phase flow in porous media [29, 30] or transport of a tracer in heterogeneous media [5, 28], the definition introduced here allows to take into account the porosity variations into the R.E.V.

$$
\left\{\varepsilon_{\beta} C_{A \beta}\right\}=\frac{1}{V_{t}} \int_{V_{t}} \varepsilon_{\beta} C_{A \beta} d V=\varepsilon_{\beta}^{*} C_{A \beta}^{*}
$$

In the same way, the core-scale intrinsic velocity $\mathbf{U}_{\beta}^{*}$ is defined as follows

$$
\left\{\mathbf{V}_{\beta}\right\}=\left\{\varepsilon_{\beta}\left\langle\mathbf{v}_{\beta}\right\rangle^{\beta}\right\}=\varepsilon_{\beta}^{*} \mathbf{U}_{\beta}^{*}=\mathbf{V}_{\beta}^{*}
$$

whereas the spatial deviations associated to the macroscopic variables presented above are introduced by

$$
\begin{aligned}
C_{A \beta} & =C_{A \beta}^{*}+\tilde{C}_{A \beta} \\
\varepsilon_{\beta}^{-1} \mathbf{V}_{\beta} & =\mathbf{U}_{\beta}^{*}+\tilde{\mathbf{U}}_{\beta}
\end{aligned}
$$


If one consider that the reference volume is very small compared to the characteristic length of the averaged property [33], it is possible to remove the macroscopic variable from the volume integral which leads to

$$
\begin{aligned}
\left\{\varepsilon_{\beta} \tilde{C}_{A \beta}\right\} & =0 \\
\left\{\varepsilon_{\beta} \tilde{\mathbf{U}}_{\beta}\right\} & =0
\end{aligned}
$$

At this point, the averaging method applied to the local transport equations leads to

$$
\begin{aligned}
\frac{\partial\left(\varepsilon_{\beta}^{*} C_{A \beta}^{*}\right)}{\partial t}+\nabla \cdot\left(\mathbf{V}_{\beta}^{*} C_{A \beta}^{*}\right)=\nabla & \cdot\left\{\mathbf{D}_{\beta}^{*}\right\} \cdot \nabla C_{A \beta}^{*}+\underbrace{\left\{\mathbf{D}_{\beta}^{*} \cdot \nabla \tilde{C}_{A \beta}\right\}}_{\text {heterogeneous dispersion }} \\
& -\underbrace{\nabla \cdot\left(\varepsilon_{\beta} \tilde{\mathbf{U}}_{\beta} \tilde{C}_{A \beta}\right)}_{\text {core-scale dispersion }}-\left\{\alpha C_{A \beta}\right\} \\
\frac{\partial \varepsilon_{\beta}^{*}}{\partial t}= & \frac{\beta}{\rho_{\sigma}}\left\{\alpha C_{A \beta}\right\}
\end{aligned}
$$

We can see here that the difficulty comes from the term $\left\{\alpha C_{A \beta}\right\}$. If it is always possible to define a core-scale mass transfer coefficient under the form,

$$
\left\{\alpha C_{A \beta}\right\}=\alpha^{*} C_{A \beta}^{*}
$$

the non-linearity does not allow to introduce simply a spatial deviation associated to the mass transfer coefficient in order to develop the closure problem.

Although it is not possible to link explicitly both scales, this study suggests, however, a plausible form of the core-scale transport-reaction equations. The terms containing the spatial deviations in Eq. (55) can be seen as some dispersion terms and a core-scale dispersive tensor $\mathbf{D}_{\beta}^{* *}$ can be defined involving these properties. From these assumptions, the following macroscopic equations are obtained

$$
\begin{aligned}
\varepsilon_{\beta}^{*} \frac{\partial C_{A \beta}^{*}}{\partial t}+\mathbf{V}_{\beta}^{*} \cdot \nabla C_{A \beta}^{*} & =\nabla \cdot\left(\mathbf{D}_{\beta}^{* *} \cdot \nabla C_{A \beta}^{*}\right)-\alpha^{*} C_{A \beta}^{*} \\
\frac{\partial \varepsilon_{\beta}^{*}}{\partial t} & =\frac{\beta}{\rho_{\sigma}} \alpha^{*} C_{A \beta}^{*}
\end{aligned}
$$


As previously for the two-equation model or the macroscopic flow equation, even if the reasons are slightly different, the direct calculation of the effective coefficients is impossible at this point without further developments which will be presented in Part II.

\section{Conclusion}

A core-scale transport-reaction model has been presented in this paper. Several approaches have been explored, based on some analogy with the macroscopic model developed at the lower scale. Two different sets of model have been investigated: a one-medium model which includes in a single "effective" medium the wormhole and the porous matrix physics, and a two-medium model, for which the wormholes are modelled separately from the porous matrix. At this point, different problems have been emphasized which can be summarized below:

- The appropriate form of the core-scale equation is still a matter of discussion, especially in the case of local non-equilibrium dissolution. However, some arguments suggest that the Darcy-scale form is a good candidate to approximate the core-scale behavior in the case of local equilibrium dissolution.

- Core-scale effective parameters are difficult to be obtained directly from solving closure problems. Their values are strongly influenced by the unit cell form, representative of the domain geometry, which is a priori unknown.

- Core-scale effective properties, if relevant, may be affected by dissolution history effects. The possibility of approximating these effects by a direct relationship with the core-scale wormhole volume fraction, $\phi_{\varpi}$, has to be investigated.

We will address these questions in Part II. Since our purpose is to investigate the different possibilities for the core-scale models, we will use the Darcy-scale simulations to obtain by spatial integration some valuable informations about their expressions and their estimations rather than to solve the closure problems with some non-representative boundary conditions. 


\section{REFERENCES}

[1] B. Bazin and G. Abdulahad, Experimental investigation of some properties of emulsified acid systems for stimulation of carbonate formations. SPE 53237, presented at the SPE Middle East Oil Show, Bahrain, 20-23 February (1999).

[2] B. Bazin, C. Roque and M. Bouteca, A laboratory evaluation of acid propagation in relation to acid fracturing: Results and interpretation. SPE 30085, presented at the Europ. Formation Damage Conf., The Hague, 15-16 May (1995).

[3] C. Beckermann, S. Ramadhyani and R. Viskanta, Natural convection flow and heat transfer between a fluid layer and a porous layer inside a rectangular enclosure. J. Heat Transfer, 109 (1987), 363-370.

[4] M.A. Buijse, Mechanisms of wormholing in carbonate acidizing. SPE 37283, presented at the SPE Int. Symposium on Oilfield Chem., Houston, 18-21 February (1997).

[5] F. Cherblanc, Etude Du Transport Miscible En Milieux Poreux Hétérogènes: Prise En Compte Du Non-Équilibre. PhD thesis, Université de Bordeaux I, (1999).

[6] F. Cherblanc, A. Ahmadi and M. Quintard, Two-medium description of dispersion in heterogeneous porous media: Calculation of macroscopic properties. Water Resources Res., 39 (6) (2003), 1154.

[7] G. Daccord, R. Lenormand and O. Lietard, Chemical dissolution of a porous medium by a reactive fluid. $1-$ model for the wormholing phenomenon. Chem. Engng. Sci., 48 (1) (1993), 169-178.

[8] C.N. Fredd, Dynamic model of wormhole formation demonstrates conditions for effective skin reduction during carbonate matrix acidizing. SPE 59537, presented at the Permian Basin Oil and Gas Recovery Conference, Texas, march (2000).

[9] C.N. Fredd and H.S. Fogler, Alternative stimulation fluids and their impact on carbonate acidizing. SPE J., 13 (1) (1998), 34.

[10] C.N. Fredd and H.S. Fogler, Influence of transport and reaction on wormhole formation in porous media. AIChE J., 44 (9) (1998), 1933-1949.

[11] C.N. Fredd and H.S. Fogler, Optimum conditions for wormhole formation in carbonate porous media: Influence of transport and reaction. SPE J., 4 (3) (1999).

[12] C.N. Fredd and M.J. Miller, Validation of carbonate matrix stimulation models. SPE 58713, presented at the Int. Symposium on Formation Damage, Lafayette (Louisiana), February (2000).

[13] T.P. Frick, M. Kurmayr and M.J. Economides, Modeling of fractal patterns in matrix acidizing and their impact on well performance. SPE 23789, presented at the SPE Production and Facilities, February (1994).

[14] R.D. Gdanski, A fundamentally new model of acid wormholing in carbonates. SPE 54719, presented at the European Formation Damage Conference, The Hague, May (1999). 
[15] F. Golfier, C. Zarcone, B. Bazin, R. Lenormand, D. Lasseux and M. Quintard, On the ability of a Darcy-scale model to capture wormhole formation during the dissolution of a porous medium. J. Fluid Mech., 457 (2002), 213-254.

[16] W.G. Gray, A derivation of the equations for multiphase transport. Chem. Engng. Sci., 30 (1975), 229-233.

[17] C.G. Groves and A.D. Howard, Early development of karst systems. 1: Preferential flow path enlargement under laminar flow. Water Resour. Res., 30 (10) (1994), 2837-2846.

[18] M.L. Hoefner and H.S. Fogler, Pore evolution and channel formation during flow and reaction in porous media. AIChE J., 34 (1) (1988), 45-54.

[19] T. Huang, A.D. Hill and R.S. Schechter, Reaction rate and fluid loss: The keys to wormhole initiation and propagation in carbonate acidizing. SPE 37312, presented at the SPE Int. Symposium on Oilfield Chem., Houston, 18-21 February (1997).

[20] T. Huang, D. Zhu and A.D. Hill, Prediction of wormhole population density in carbonate matrix acidizing. SPE 54723, presented at the Europ. Formation Damage Conf., The Hague, may 31-june 1 (1999).

[21] E.J. Koval, A methor for predicting the performance of unstable miscible displacement in heterogeneous porous media. Trans. AIME, 228 (1963), 145-154.

[22] D. Lasseux, F. Golfier, M. Quintard and H. Bertin, On the determination of effective permeability of vuggy porous media. In: 2nd Int. Conf. On Computer Methods for Engineering in Porous Media, Besançon, Jul. (2000).

[23] X. Liu, A. Ormond, K. Bartko, Y. Li and P. Ortoleva, A geochemical reaction-transport simulator for matrix acidizing analysis and design. J. of Pet. Sci. and Engng., 17 (1997), 181-196.

[24] K. Lund, H.S. Fogler and C.C. McCune, The dissolution of dolomite in hydrochloric acid. Chem. Engng Sci., 28 (1973), 691.

[25] K. Lund, H.S. Fogler, C.C. McCune and J.W. Ault, The dissolution of calcite in hydrochloric acid. Chem. Engng. Sci., 30 (1975), 825.

[26] C. Moyne, S. Didierjean, H.P. Amaral Souto and O.T. da Silveira, Dispersion thermique en milieu poreux: Modèle à une équation. In: Congrès Annuel de la Société Française des Thermiciens, pages 467-472. Elsevier, (1999).

[27] M. Quintard, One-phase flow in vuggy rocks. Internal report of L.E.P.T.-ENSAM, December (1996).

[28] M. Quintard, F. Cherblanc and S. Whitaker, Dispersion in heterogeneous porous media: One-equation non-equilibrium model. Transport in Porous Media, 44 (1) (2001), 181-203.

[29] M. Quintard and S. Whitaker, Two-phase flow in heterogeneous porous media: The method of large-scale averaging. Transport in Porous Media, 3 (1988), 357-413. 
[30] M. Quintard and S. Whitaker, Two-phase flow in heterogeneous porous media: The influence of large spatial and temporal gradient. Transport in Porous Media, 5 (1990), 341-379.

[31] M. Quintard and S. Whitaker, One and two-equation models for transient diffusion processes in two-phase systems. Adv. Heat Transfer, 23 (1993), 369-464.

[32] M. Quintard and S. Whitaker, Transport in ordered and disordered porous media: Volume averaged equations, closure problems and comparison with experiment. Chem. Engng. Sci., 48 (1993), 2537-2564.

[33] M. Quintard and S. Whitaker, Transport in ordered and disordered porous media I: The cellular average and the use of weighting functions. Transport in Porous Media, 14 (1994), $163-177$.

[34] M. Quintard and S. Whitaker, Local thermal equilibrium for transient heat conduction: Theory and comparison with numerical experiments. In: J. Heat Mass Transfer, 38 (1995), 2779-2796.

[35] M. Quintard and S. Whitaker, Transport in chemically and mechanically heterogeneous porous media - III. Large-scale mechanical equilibrium and the regional form of Darcy's law. Adv. in Water Resour., 21 (1998), 617-629.

[36] M. Quintard and S. Whitaker, Dissolution of an immobile phase during flow in porous media. Ind. and Engng. Chem. Res., 38 (3) (1999), 833-844.

[37] G. Rowan, Theory of acid treatment of limestone formations. J. Inst. Petrol., 45 (431) (1959).

[38] M.A.A. Spaid and F.R. Phelan Jr., Lattice Boltzmann methods for modeling microscale flow in fibrous porous media. Phys. Fluids, 9 (9) (1997), 2468-2474.

[39] K. Vafai and R. Thiyagaraja, Analysis of flow and heat transfer at the interface region of a porous medium. Int. J. Heat Mass Transfer, 30 (7) (1987), 1391-1405.

[40] Y. Wang, A.D. Hill and R.S. Schechter, The optimum injection rate for matrix acidizing of carbonate formations. SPE 26578, presented at the Tech. Conf. and Exhib., Houston, (1993).

[41] Z.F. Zhang and J.E. Smith, The velocity of DNAPL fingering in water-saturated porous media: Laboratory experiments and a mobile-immobile model. J. of Contaminant Hydrology, 49 (2001), 335-353. 